ORIGINAL ARTICLE

\title{
The Impact of Preemptive Perianal Anaesthetic Block in Open Haemorrhoidectomy - a randomised controlled trial
}

\author{
WAJEEHA IMRANI ANDRABI, MOHAMMAD ASADULLAH KHAWAJA, K FATIMA, SYED IMRAN HUSSAIN ANDRABI, A \\ SHAFIQUE, SHAUKAT RABBANI, SAQIB ZAHUR, HASNAT AHMAD BUTT, ABDUL MAJEED CHAUDHRY \\ *Department of Radiology, Doctors Hospital, Lahore / Ghurki Trust Teaching Hospital, Lahore, Pakistan \\ Department of Surgery, Ghurki Trust Teaching Hospital, Lahore, Pakistan \\ Correspondence to Prof. Syed Imran Hussain Andrabi, Professor of Surgery, Email: imranandrabi@gmail.com, Te.+923344478999
}

\begin{abstract}
Background: the study was conducted to analyze the efficacy of $0.75 \%$ ropicaine at perianal block for open haemrrhoidectomy with regards to pain intensity, first demand of analgesia and hospital stay.

Method: 50 patients were selected for open haemrrhoidectomy under GA which were divided into two groups.it was a randomized control trial in which sealed envelope method was used for the group delegation in which Group A was designated to the patients having the perianal block with $0.75 \%$ ropivacaine while the group $B$ was the placebo group having normal saline injected in the perianal region. The variables compared were the first demand of analgesia, pain intensity and the duration of the hospital stay. In order to make the site of injection more authentic the injections were sited under ultrasound guidance.

Results: The pain intensity which was analyzed with the visual analogue score (VAS) had a median of 3.8 (high=6, low=3) in group A while 5.5 (high=8, low=4) in the Group B with the p value of $<0.05$. The mean time recorded for the first demand of analgesia was $6.20 \pm 1.20$ hours in the Group A which had improved from $1.20 \pm 1.0$ hours in the Placebo Group while the p value was $<0.001$, while the mean time of hospital stay got reduced from $22.5 \pm 3.30$ hours to $12.4 \pm 3.10$ hours with the significant $p$ value of $<0.002$.

Conclusion: It was observed that with preemptive analgesia with $0.75 \%$ ropivacaine administered led to a substantial reduction in pain perception, request for an analgesic and hospital stay. Therefore it is appropriate to administer it before open hemorrhoidectomy.

Keywords: preemptive anaesthesia, local anesthesia, postoperative pain, ropivacaine, open hemorrhoidectomy
\end{abstract}

\section{INTRODUCTION}

Five percent of the adult people present with anorectal diseases to the hospitals ${ }^{1}$. Open hemorrhoidectomy is one of the most common procedures performed in general surgery ${ }^{1}$. Postoperatively, effective pain control has a major influence on post operative recovery and the hospital stay ${ }^{2}$.

Post operative pain in open haemrrhoidectomy is a major concern due to which various analgesic options and routes have been contemplated. Perianal ring block which is a form of loco-regional anesthesia has also been explored in this regard. It has proved to be a very promising option in postoperative pain management ${ }^{4}$ and even it has been reported to be as effective as a caudal block ${ }^{5}$. Administering the loco-regional anesthesia not only decreases the intensity of postoperative pain ${ }^{6}$ but also cuts down hospital stay and the cost of treatment ${ }^{7,8}$.

The objective of our trial is to validate the efficacy of perianal blocks based on three variables one of which is reducing the postoperative pain, secondly the time taken for first demand of analgesia after the procedure and thirdly was the duration of the hospital stay following the procedure.

\section{METHODS}

This study was conducted between Jan 2017 to Jan 2021 at Department of Surgery, Ghurki Trust Teaching Hospital, Jallo Mor, Lahore, Pakistan after approval from Ethical Committee. The inclusion criteria was all the ASA I

Received on 27-04-2021

Accepted on 17-08-2021 and II category patients between the age of 20-60 years. 50 patients were randomly allocated by the closed envelope method technique in which Group A (Ropivacaine group) was allocated to 25 and the Group B (the placebo Group) to the other 25.

The patients having any other perianal pathology along with Haemrrhoids or morbidly obese with the BMI > 30 were excluded. All the patients were administered general anesthesia and underwent an open hemorrhoidectomy.

After an informed consent, all surgeries were performed in lithotomy position by experienced Consultant Surgeons (at least after five years of post-graduation). The nerve block was administered under ultrasound guidance in the first four patients so as to bring more anatomical authenticity to the study and for better results. After that all the patients were managed without the sonographic expertise. Selected patients were randomly assigned to one of the two groups. Group $1(n=25)$ patients received perianal block with $20 \mathrm{ml}$ of $0.75 \%$ Ropivacaine while patients of Group $2(n=25)$ received $20 \mathrm{ml}$ of isotonic saline infiltration. It was administered circumferentially in and around the anal sphincter and submucosally through punctures at 3, 6, 9 and 12 o' clock loci. The objective was to target the terminal branches of the anococcygeal, puedendal, inferior rectal and perineal nerves (Figure 1). Ultrasound guidance permitted precise placement of local anesthetic. Prophylaxis was insinuated with Intravenous ciprofloxacin $200 \mathrm{mgs}$ and metronidazole $500 \mathrm{mgs}$ and these medications were continued orally for next 3 days. Standard Millighan Morgan Hemorrhoidectomy was performed. 
Analgesia was planned with Paracetamol 1000mg at the first demand of analgesia while break through pain was managed with intra venous Ketorolac $30 \mathrm{mg}$. The pain intensity was calculated with the Visual Analogue Score ranging from 0 that was no pain to 10 being the maximum. The second factor of the first demand of analgesia was time duration measured from the time of surgery to the time of administration of analgesia on the demand of the patient which should be more than 3 on VAS. The length of the hospital stay was also taken into account as the third factor being measured. On discharge all the patients were prescribed Paracetamol 500mg three times daily alternating with lbuprofen $400 \mathrm{mg}$ along with a stool softener and the patient was advised to have sitz bath after each defecation.

All Group 1 patients were discharged home after the ward round the next morning. Most Group 2 patients stayed on for another 24 hours with unsettled pain.

Figure 1: Anatomy of Perianal area courtesy Delaney CP. Netter's surgical anatomy and approaches. Elsevier Health Sciences; 2013 Jul 2.

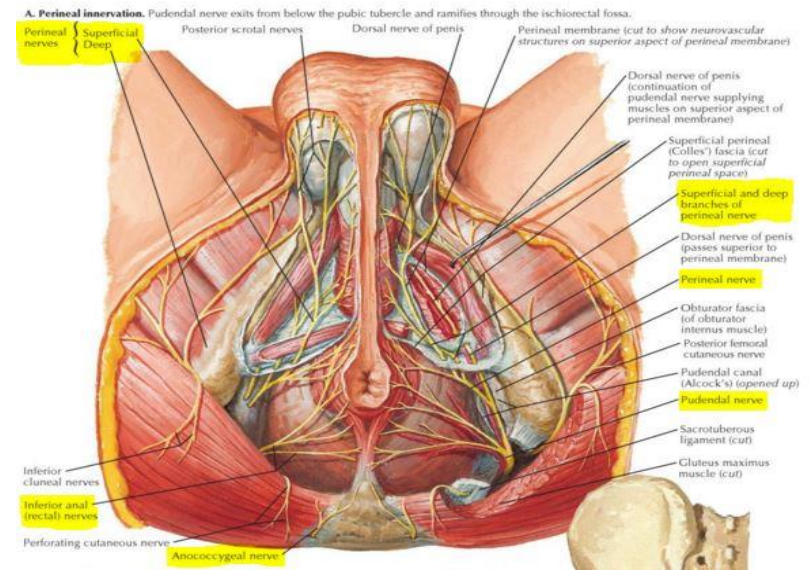

Statistical analysis: SPSS 22 was used for the statistical analysis. $P$ value of $<0.05$ was considered significant while mean and median of all the vaiables were also calculated.

\section{RESULTS}

The study included 50 patients in which Group A $(n=25)$ had a perianal block with $20 \mathrm{ml}$ of $0.75 \%$ Ropivaciane while Group B ( $n=25)$ was the placebo group with the $20 \mathrm{ml}$ of isotonic saline infiltration. There was no significant difference in the age between the two groups (Table 1).

Pain intensity was assessed at the first demand of analgesia in which the Median of VAS score of group A was 3.8 with a range of 3-6, where 3 being the lowest while 6 being the highest, while for the group $B$ the median was 5.5 with a range of $4-8$ with the $p$ value of $<0.05$ (Table 1 ).

The mean time for the demand of first dose of analgesia for group 1 was $6.20 \pm 1.20$ hours and group 2 was $1.20 \pm 1.0$ hours with the $p$ value of $<0.001$ (Table 1 ).

Mean hospital stay for group 1 was $12.4 \pm 3.10$ hours while for group 2 was $22.5 \pm 3.30$ hours $p$ value $<0.002$ (Table 1).
Table 1

\begin{tabular}{|l|c|c|c|}
\hline Parameter & Group 1 & Group 2 & P-value \\
\hline Age & $44.7 \pm 10.5$ & $40.1 \pm 12.2$ & 0.5 \\
\hline Analgesia required & $6.2 \pm 1.2$ & $1.2 \pm 1.0$ & 0.001 \\
\hline Hospital stay & $12.4 \pm 3.1$ & $22.5 \pm 3.3$ & 0.002 \\
\hline VAS & $3.8 \pm 1.5$ & $5.5 \pm 2.3$ & 0.05 \\
\hline
\end{tabular}

\section{DISCUSSION}

Hemorrhoidectomy is a common surgical procedure. It leads to severe postoperative pain. It culminates to anguish and apprehension. The pain management is one of the most cumbersome aspects of this procedure which is always in the back of the mind of the operating surgeon. Many methodologies have been adopted keeping the pain ladder in mind but still the requirement of analgesia is very high post operatively. It has been postulated that not only breaking the pain cycle but also inhibiting the initiation of this cycle is a more profound strategy. Studies have shown that the local nerve blocks and the site of surgery being infiltrated by the local anesthesia have also shown a lot of promise in this aspect ${ }^{8}$. This observation prompted this study.

Current international trend are inclined towards day case surgery for anorectal diseases ${ }^{9}$. As mentioned earlier that the infiltration of local anaesthetics and local nerve blocks for the post operative pain management in anorectal surgeries have immensely aided this trend ${ }^{6}$. This is the day and age of ERAS Early recovery after surgery where the aim is for an early discharge of the patients which is only possible under trained surgeons and an even more effective post operative pain management that makes the an early discharge possible. For this reason we resorted to strategy of preemptive anaesthesia. We observed that the Group A receiving ropivacaine showed not only a significant decrease in pain perception but also the duration of the first demand of analgesia was also increased hence leading onto an early discharge. On the other hand the Group B receiving placebo showed a longer hospital stay because their pan score according to the VAS was higher than the group $A$ and a shorter duration of the first demand of analgesia. There is going to be a positive impact on the affordability of the patient by lifting a substantial financial burden, owing to the fact the majority of our patients belong to low socio economic status of the society.

There are many techniques of administering perianal block. The local anaesthesia can be infiltrated into the sphincter [10] and in the ischiorectal fossae ${ }^{11}$. This technique has three variants. Infiltration posteriorly alone ${ }^{12}$, both anteriorly \& posteriorly ${ }^{9}$, and all around the sphincter complex ${ }^{13}$. These techniques have been combined with infiltration into the intersphincteric space ${ }^{14}$, submucosal space and perianal skin ${ }^{15}$. We used a combination of all the above. To make it more effective we used the ultrasound guidance in the first four cases.

Primarily $0.75 \%$ ropicaine was used. Sometimes ropivacaine ${ }^{11}$ alone or in a combination of lignocaine or bupivacaine, both with and without adrenaline ${ }^{1}$ were used. In our patients pain relief lasted 12 hrs, in accord with experience of others ${ }^{12}$. Moreover, pain relief for up to 24 hours has been also stated ${ }^{16}$. The Group A receiving preemptive ropivacaine showed significant reduction in the median value of VAS. They also showed a considerable 
increase in the duration of the first demand of analgesia, hence alleviating the stress and anxiety of the patient. Moreover the patient was mobilized earlier and leading to an early discharge. In comparison Group B receiving placebo had an exaggerated pain response with a higher median value of VAS and also a lower median value for the first demand of analgesia and increased hospital stay as observed in the results. This observation is consistent with earlier studies ${ }^{16}$.

A worrying complication of anorectal surgery is urinary retention. It can occur in up to $7 \%-17 \%$ of patients ${ }^{13,17}$. Perineal pain followed by prolonged autonomic blockade, excessive intravenous fluids and limited movement can lead to urinary retention [13]. Perianal block reduces urinary retention by relieving perineal pain and allowing early mobilization. No patients suffered from urinary retention in our study.

These results have led us to bring about certain policy alterations in theatres. We have made sure the presence of local anaesthesia on the instrument trolley.

This study was conducted in a hospital situated at the peripheral border area of the city which is thinly populated, subsequently leading to a small sample size. Studies with bigger sample sizes should be performed to strengthen the proposal.

In Summary, we have found preemptive perianal block for open hemorrhoidectomy, as safe, easy to administer, quick recovery, speedy discharge, good postoperative pain relief and low cost. These benefits validate the acceptance of perianal block for open hemorrhoidectomy.

\section{CONCLUSION}

Perianal nerve blocks with local infiltration with ropivacaine is effective in managing pain following open hemorrhoidectomy. It is apparent from our study that this technique considerably decreases postoperative pain perception and analgesic requirement, thus reducing the hospital stay. As this study has convinced us to use it routinely in our existing practice, we would endorse the same for our international contemporaries.

Funding: None.

Conflict of interests: None

\section{REFERENCES}

1. S. Li, M. Coloma, P.F. White, M.F. Watcha, J.W. Chiu, H. Li, et al., Comparison of the costs and recovery profiles of three anesthetic techniques for ambulatory anorectal surgery., Anesthesiology. $93 \quad$ (2000) 1225-1230. doi:10.1097/00132586-200108000-00002.

2. A. Kazemeini, M. Rahimi, M.S. Fazeli, S.A. Mirjafari, H. Ghaderi, K. Fani, et al., The effect of local injections of bupivacaine plus ketamine, bupivacaine alone, and placebo on reducing postoperative anal fistula pain: A randomized clinical trial, Sci. World J. 2014 (2014). doi:10.1155/2014/424152.

3. S.F. Hon, C.M. Poon, H.T. Leong, Y.C. Tang, Pre-emptive infiltration of Bupivacaine in laparoscopic total extraperitoneal hernioplasty: A randomized controlled trial, Hernia. 13 (2009) 53-56. doi:10.1007/s10029-008-0422-9.

4. R. Gerjy, A. Lindhoff-Larson, R. Sjödahl, P.O. Nyström, Randomized clinical trial of stapled haemorrhoidopexy performed under local perianal block versus general anaesthesia, Br. J. Surg. 95 (2008) 1344-1351. doi:10.1002/bjs. 6379 .

5. J. Gudaityte, I. Marchertiene, D. Pavalkis, Anesthesia for ambulatory anorectal surgery., Medicina (Kaunas). 40 (2004) 101-111.

6. K.S. Ho, K.W. Eu, S.M. Heah, F. Seow-Choen, Y.W. Chan, Randomized clinical trial of Haemorrhoidectomy under a mixture of local anaesthesia versus general anaesthesia, $\mathrm{Br}$. J. Surg. 87 (2000) 410-413. doi:10.1046/j.13652168.2000.01411.x.

7. I.T. Awad, F. Chung, Factors affecting recovery and discharge following ambulatory surgery, Can. J. Anesth. 53 (2006) 858-872. doi:10.1007/BF03022828.

8. C.K.-S. Ong, P. Lirk, R. a Seymour, B.J. Jenkins, The efficacy of preemptive analgesia for acute postoperative pain management: a meta-analysis., Anesth. Analg. 100 (2005) 757-773, table of contents. doi:10.1213/01.ANE.0000144428.98767.0E.

9. M.C. Marti, B. Roche, Outpatient proctological surgery, Ambul. Surg. 6 (1998) 25-28. doi:10.1016/S09666532(97)10010-5.

10. P.J. Gupta, Feasibility of day care surgery in proctology, J. Gastrointest. Liver Dis. 15 (2006) 359-362. doi:5 [pii].

11. P.O. Nyström, K. Derwinger, R. Gerjy, Local perianal block for anal surgery, Tech. Coloproctol. 8 (2004) 23-26. doi:10.1007/s10151-004-0046-8.

12. F. Gabrielli, U. Cioffi, M. Chiarelli, A. Guttadauro, M. De Simone, Hemorrhoidectomy with posterior perineal block: experience with 400 cases, Dis Colon Rectum. 43 (2000) 809-812.

http://www.ncbi.nlm.nih.gov/entrez/query.fcgi?cmd=Retrieve\& $\mathrm{db}=$ PubMed\&dopt=Citation\&list_uids $=10859082$.

13. R. Kushwaha, W. Hutchings, C. Davies, N.G. Rao, Randomized clinical trial comparing day-care open haemorrhoidectomy under local versus general anaesthesia, in: Br. J. Surg., 2008: pp. 555-563. doi:10.1002/bjs.6113.

14. Z. Kazak, P. Ekmekci, K. Kazbek, Hyperbaric levobupivacaine in anal surgery: Spinal perianal and spinal saddle blocks, Anaesthesist. 59 (2010) 709-713. doi:10.1007/s00101-010-1755-1.

15. F.S.P. Regadas, S.M.M. Regadas, L. V. Rodrigues, R. Misici, F.R. Silva, F.S.P. Regadas Filho, Transanal repair of rectocele and full rectal mucosectomy with one circular stapler: A novel surgical technique, Tech. Coloproctol. 9 (2005) 63-66. doi:10.1007/s10151-005-0197-2.

16. A.J. Luck, P.J. Hewett, Ischiorectal fossa block decreases posthemorrhoidectomy pain: randomized, prospective, double-blind clinical trial., Dis. Colon Rectum. 43 (2000) 142 5. http://www.ncbi.nlm.nih.gov/pubmed/10696885.

17. H.O. Chin, E.C.B. Foo, V. Keng, Ambulatory circular stapled haemorrhoidectomy under local anaesthesia versus circular stapled haemorrhoidectomy under regional anaesthesia, ANZ J. Surg. 75 (2005) 184-186. doi:10.1111/j.14452197.2005.03330.x 